\title{
Twin studies on the effect of genetic factors on serum agalactosyl immunoglobulin G levels
}

\author{
KANAKO AZUMA $^{1 *}$, SHINICHIRO SHINZAKI $^{1 *}$, HITOMI ASAZAWA $^{1 *}$, ERI KUROKI $^{1}$, \\ SAYURI KAWAMOTO ${ }^{1}$, YOSHIHIRO KAMADA ${ }^{1}$, KAZUO HAYAKAWA $^{2}$ and EIJI MIYOSHI ${ }^{1}$ \\ Departments of ${ }^{1}$ Molecular Biochemistry and Clinical Investigation and ${ }^{2}$ Health Promotion Science, \\ Osaka University Graduate School of Medicine, Suita, Osaka 565-0871, Japan
}

Received November 5, 2013; Accepted December 20, 2013

DOI: $10.3892 /$ br.2014.216

\begin{abstract}
The level of immunoglobulin G (IgG) lacking the terminal galactose, referred to as agalactosyl $\mathrm{IgG}$, was found to be increased in chronic inflammatory diseases, such as rheumatoid arthritis and inflammatory bowel disease (IBD), particularly in Crohn's disease, which is suggested to have a genetic component. This oligosaccharide modification of IgG is mainly regulated by the expression of glyco-genes; however, the association between genetic factors and changes in the IgG glycosylation has not been fully elucidated. The aim of the present study was to assess the role of genetics in this process by comparing the serum agalactosyl IgG levels between members of monozygotic and dizygotic twin pairs who underwent medical check-ups at the same time. The serum agalactosyl IgG level was assayed using high-performance liquid chromatography. Hematological and biochemical markers, including $\gamma$-glutamyltranspeptidase $(\gamma \mathrm{GTP})$, alanine aminotransferase (ALT) and white blood cell (WBC) count, were also measured. Although the serum $\gamma$ GTP levels (and, to a lesser extent, ALT and WBC levels) exhibited a correlation within monozygotic twin pairs, agalactosyl IgG levels were not found to be correlated between members of either type of twin pairs. Thus, the role of genetic factors in determining serum agalactosyl IgG levels may be less significant compared to the effect of environmental factors or the onset of inflammatory disease.
\end{abstract}

Correspondence to: Professor Eiji Miyoshi, Department of Molecular Biochemistry and Clinical Investigation, Osaka University Graduate School of Medicine, 1-7 Yamada-oka, Suita 565-0871, Osaka, Japan

E-mail: emiyoshi@sahs.med.osaka-u.ac.jp

*Contributed equally

Abbreviations: IgG, immunoglobulin G; IBD, inflammatory bowel disease

Key words: agalactosyl immunoglobulin G, environmental factors, genetic factors, monozygotic twin pairs, dizygotic twin pairs

\section{Introduction}

Immunoglobulin $\mathrm{G}(\mathrm{IgG})$ possesses complex-type biantennary $N$-linked oligosaccharides at asparagine 297 of the $\mathrm{C} \gamma 2$ domain of the Fc fragment (1). Some of these oligosaccharides have bisecting $N$-acetylglucosamine (GlcNAc), core-fucose, galactose and sialic acid residues (2,3). Patients with rheumatoid arthritis (4) and other chronic inflammatory diseases, such as systemic lupus erythematosus, Sjogren's syndrome and tuberculosis $(5,6)$, exhibit elevated serum levels of agalactosyl IgG, an IgG oligosaccharide that lacks the terminal galactose. We recently reported that serum agalactosyl $\mathrm{IgG}$ levels may be a novel diagnostic marker for the activity and clinical course of inflammatory bowel disease (IBD) (7) and developed a method to determine agalactosyl IgG using a lectin-antibody ELISA (8). Furthermore, we demonstrated the pathophysiological role of agalactosyl IgG in IBD using a mouse model of experimental colitis that is deficient in $\beta-1,4$-galactosyltransferase (9). Those experiments indicated that the increase in agalactosyl $\mathrm{IgG}$ levels in patients with IBD may be associated with the host's defense against inflammation, rather than the etiology of IBD.

We previously evaluated the levels of agalactosyl IgG by measuring the ratio of agalactosylated to fucosylated IgG oligosaccharides (G0F/G2F) (7) and demonstrated that $\mathrm{G} 0 \mathrm{~F} / \mathrm{G} 2 \mathrm{~F}$ is a marker of IBD clinical activity and prognosis of recurrence. However, some patients with Crohn's disease do not exhibit elevated agalactosyl IgG levels, despite severe disease activity, suggesting that genetic factors may dictate IgG galactosylation. Furthermore, the level of IgG agalactosylation was shown to increase with age (10) and may be regulated by a variety of environmental factors, including food and infection; therefore, the relative effect of genetic and environmental factors has not been clearly determined. To determine the effect of genetic factors on the agalactosylation of $\mathrm{IgG}$, we investigated the correlations of $\mathrm{G} 0 \mathrm{~F} / \mathrm{G} 2 \mathrm{~F}$ and other biochemical data within pairs of monozygotic and dizygotic twins who underwent simultaneous medical check-ups.

\section{Materials and methods}

Subjects. The characteristics of the participants are summarized in Table I. Sixteen monozygotic twin pairs (14 males and 18 females, aged $40.8 \pm 19.3$ years) and 13 dizygotic twin pairs 
A

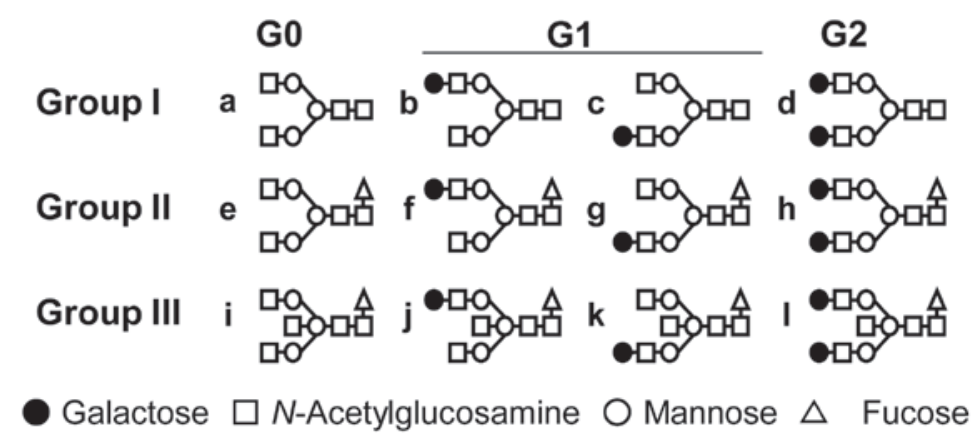

B

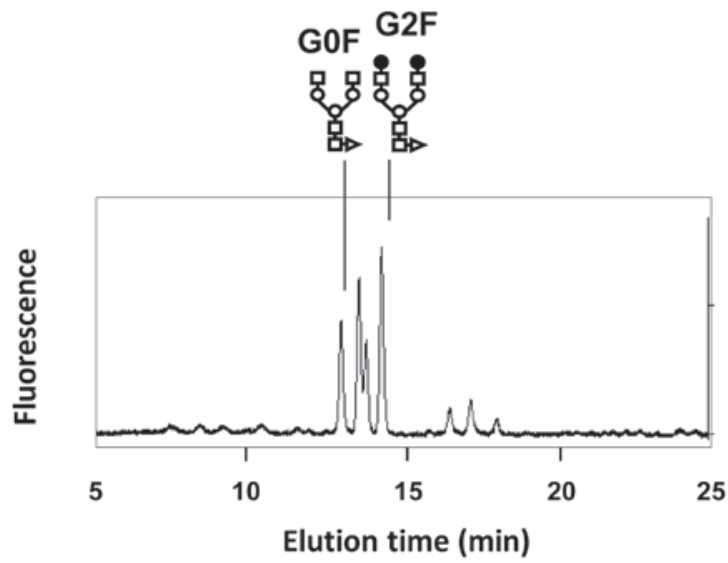

Figure 1. Analysis of 2pyridylamino (PA)-labeled IgG oligosaccharides with high-performance liquid chromatography. (A) Structural patterns of N-linked neutral oligosaccharides on IgG. (B) Representative profiles of 2PA-labeled oligosaccharides derived from IgG under neutral conditions.

$\mathbf{A}$

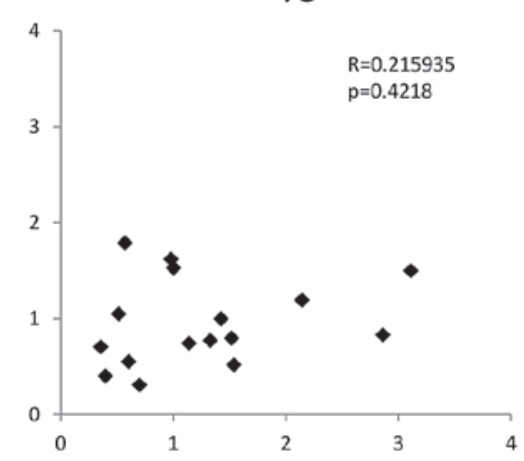

Monozygotic twins

\section{B}

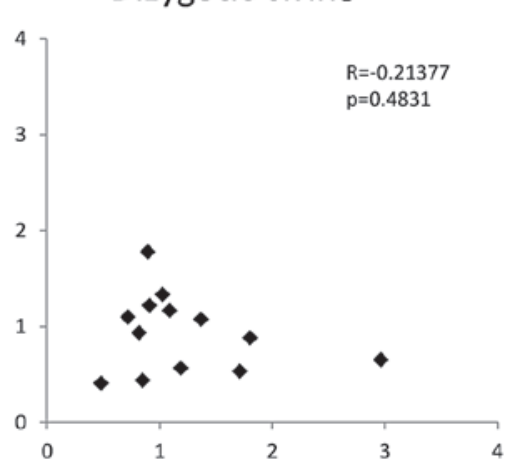

C

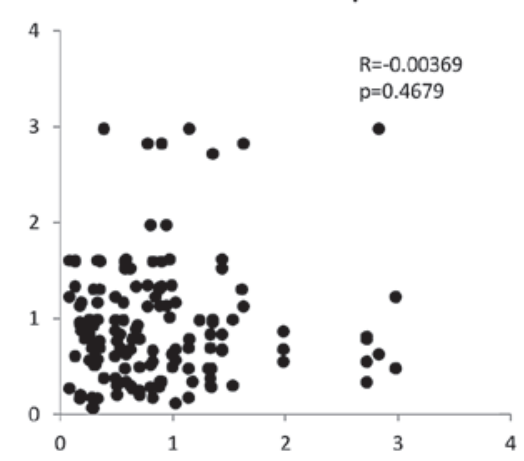

Figure 2. Scatterplots of G0F/G2F ratios for (A) monozygotic twins; (B) dizygotic twins; and (C) unrelated pairs. In (A) and (B), the higher G0F/G2F ratio within the pair was plotted on the horizontal axis.

Table I. Subject participant characteristics (means \pm SD).

\begin{tabular}{lcc}
\hline Characteristics & $\begin{array}{c}\text { Monozygotic } \\
\text { twins }\end{array}$ & $\begin{array}{c}\text { Dizygotic } \\
\text { twins }\end{array}$ \\
\hline Pairs (n) & 16 & 13 \\
Male/female & $14 / 18$ & $10 / 16$ \\
Age (years) & $40.8 \pm 19.3$ & $42.5 \pm 16.9$ \\
$\gamma$-glutamyltranspeptidase (IU/l) & $25.4 \pm 25.7$ & $22.8 \pm 35.4$ \\
Alanine aminotransferase (IU/l) & $16.8 \pm 9.01$ & $14.8 \pm 9.30$ \\
White blood cells/ $\mu$ 1 & $5,909 \pm 1,819$ & $5,276 \pm 1,505$ \\
G0F/G2F ratio & $1.10 \pm 0.68$ & $1.07 \pm 0.55$ \\
\hline
\end{tabular}

(10 males and 16 females, aged $42.5 \pm 16.9$ years) who underwent simultaneous medical check-ups as pairs between 1984 and 1994 were enrolled in this study. All the participants were healthy. Written informed consent was obtained from each subject and the study protocol was approved by the Ethics Committee of Osaka University. We also randomly selected unrelated pairs from this pool of participants and a total of 145 unrelated pairs were analyzed to serve as controls for genetic association.

IgG purification. Serum IgG was purified using protein $\mathrm{G}$ sepharose (Amersham Pharmacia Biotech, Buckinghamshire, 
A үGTP
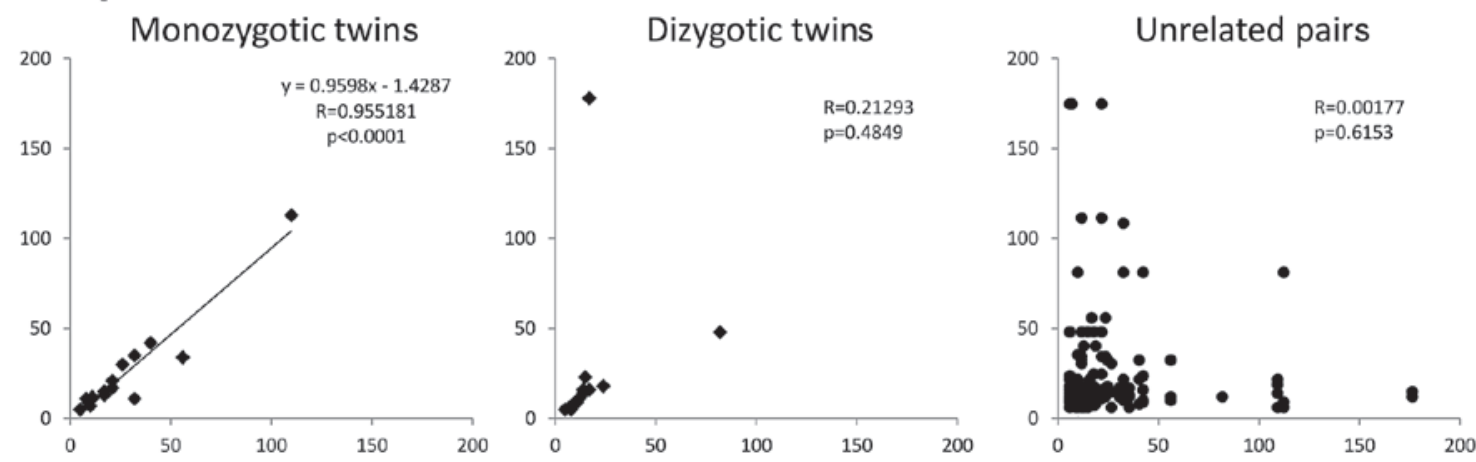

B ALT

Monozygotic twins

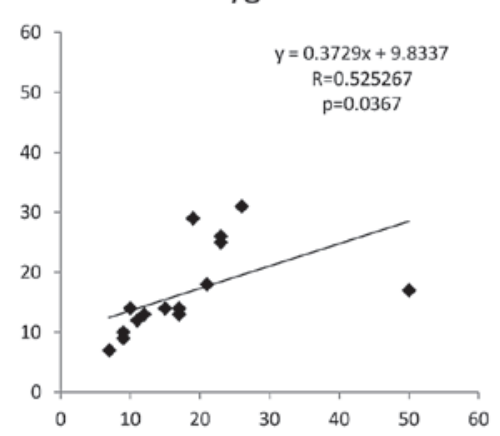

Dizygotic twins

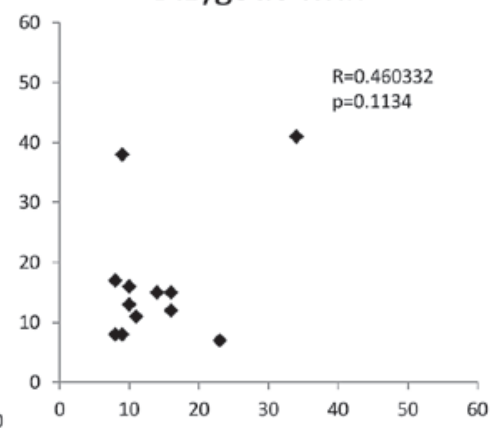

\section{Unrelated pairs}

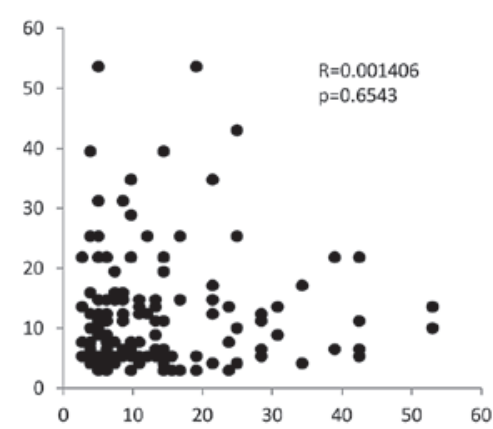

C

WBC

Monozygotic twins
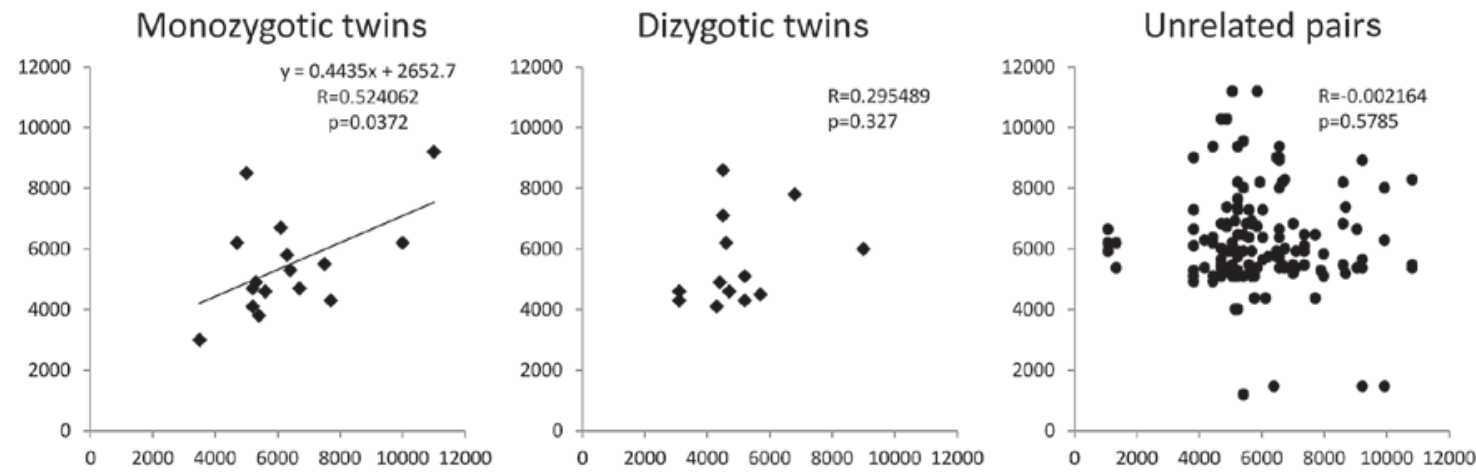

Figure 3. Scatterplots of serum levels of (A) $\gamma$-glutamyltranspeptidase ( $\gamma$ GTP); (B) alanine aminotransferase (ALT); and (C) white blood cell (WBC) count for monozygotic twins, dizygotic twins and unrelated pairs.

UK). Briefly, serum diluted 1:1 with phosphate-buffered saline (PBS) was loaded onto a protein G sepharose column. The column was subsequently washed with a minimum of 10 column volumes of PBS, followed by the same volume of $10 \mathrm{mM}$ ammonium bicarbonate. Column-bound IgG was eluted using $0.1 \%$ trifluoroacetic acid.

Analysis of IgG oligosaccharides. The pyridylaminated $\mathrm{N}$-linked oligosaccharide of $\mathrm{IgG}$ was analyzed using reverse-phase high-performance liquid chromatography (HPLC). $N$-linked oligosaccharides were released from serum IgG and labeled with 2-aminopyridine as previously described (7). Briefly, $N$-linked oligosaccharides were released from purified $\mathrm{IgG}$ samples following overnight incubation with $0.5 \mathrm{mU}$ glycopeptidase F (Takara Bio, Inc., Sigma, Japan) at $37^{\circ} \mathrm{C}$. The oligosaccharides were then incubated with $0.5 \mathrm{mM}$ ammonium acetate $(\mathrm{pH} \mathrm{4.0)}$ for $30 \mathrm{~min}$, lyophilized and labeled with 2-aminopyridine using GlycoTag (Takara Bio, Inc.) according to the manufacturer's instructions. Excess reagent was removed with a cellulose cartridge glycan preparation kit (Takara Bio, Inc.) and the oligosaccharides were incubated with $2 \mathrm{M}$ acetic acid at $80^{\circ} \mathrm{C}$ for $2 \mathrm{~h}$ to remove sialic acids. The pyridylamino (PA)-oligosaccharides from IgG were analyzed with reverse-phase HPLC (Hitachi High-Technologies Corporation, Tokyo, Japan) using a LaChrom Ultra C18 $(2-\mu \mathrm{m})$ column (Hitachi High-Technologies Corporation) with $10 \mathrm{mM}$ sodium phosphate ( $\mathrm{pH} 4.4$, solvent $\mathrm{A}$ ) and $10 \mathrm{mM}$ sodium phosphate plus $0.5 \%$ 1-butanol (solvent B) at a flow rate of $0.5 \mathrm{ml} / \mathrm{min}$ at $40^{\circ} \mathrm{C}$. The glycans were separated with a gradient of $0-50 \%$ solvent B for $30 \mathrm{~min}$, followed by $50 \%$ solvent B for $10 \mathrm{~min}$. The PA-oligosaccharides were detected using a fluorescence detector (LaChrom Elite, Hitachi) at wavelengths of $320 \mathrm{~nm}$ for excitation and $400 \mathrm{~nm}$ for emission. 
Statistical analysis. The patient characteristics are presented as mean $\pm \mathrm{SD}$. The Spearman's rank correlation coefficient was used to assess the correlation of continuous variables within each pair. $\mathrm{P}<0.05$ was considered to indicate a statistically significant difference.

\section{Results}

IgG oligosaccharide profiles. The normal oligosaccharide structures of neutral human $\operatorname{IgG}$ contain 12 major structural variants (Fig. 1A). We analyzed the profiles of IgG neutral oligosaccharides using HPLC in combination with fluorescent labeling of oligosaccharides. In our previous study (7), the G0F/G2F ratio was described as the ratio of the peak height of G0 (agalactosylated IgG) to G2 (fucosylated IgG oligosaccharide group II) (Fig. 1B). Since the majority of IgG oligosaccharides belong to group II, the $\mathrm{G} 0 \mathrm{~F} / \mathrm{G} 2 \mathrm{~F}$ ratio represents the total agalactosylation of $\mathrm{IgG}$.

G0F/G2F ratio. We measured the G0F/G2F ratio of IgG oligosaccharides in 32 monozygotic and 26 dizygotic twin pairs. The G0F/G2F ratio was not found to be significantly correlated within monozygotic twin $(\mathrm{R}=0.215935)$, dizygotic twin $(\mathrm{R}=-0.21377)$, or unrelated pairs $(\mathrm{R}=-0.0369)($ Fig. 2A-C).

Correlations of different markers within pairs. The correlations in serum $\gamma$-glutamyltranspeptidase $(\gamma \mathrm{GTP})$ levels were higher within monozygotic twin $(\mathrm{R}=0.955181)$ compared to those within dizygotic twin $(\mathrm{R}=0.21293)$ and unrelated pairs ( $\mathrm{R}=0.00177)$ (Fig. 3A). Alanine aminotransferase levels ( $\mathrm{R}=0.525267$ for monozygotic, $\mathrm{R}=0.460332$ for dizygotic and $\mathrm{R}=0.001406$ for unrelated pairs) and white blood cell (WBC) count $(\mathrm{R}=0.524062$ for monozygotic, $\mathrm{R}=0.295489$ for dizygotic and $\mathrm{R}=-0.002164$ for unrelated pairs) did not exhibit a strong correlation within twin pairs, although both were found to be significant in monozygotic twin pairs $(\mathrm{P}=0.0367$ and $\mathrm{P}=0.0372$, respectively) (Fig. 3B-C).

\section{Discussion}

The agalactosylation of IgG increases with age and is associated with a number of inflammatory diseases. Although the present study included a limited number of twin pairs, the results clearly demonstrated that $\operatorname{IgG}$ agalactosylation was not significantly affected by genetics. Of note, $\gamma$ GTP levels were found to be significantly correlated in the 16 pairs of monozygotic twins investigated. Since $\gamma$ GTP levels are often associated with alcohol consumption, this finding suggests that taste and metabolism of alcohol are associated with genetic factors. Although the WBC count is known to vary under different conditions, it was similar between the monozygotic twins in this study. Therefore, compared to WBC, the agalactosylation of IgG appears to be less affected by genetic and more by environmental factors. Furthermore, our studies indicated that twin studies may not a suitable approach to glycobiology investigations.

As the HPLC analysis of IgG oligosaccharides is costly and time-consuming, high-throughput systems, such as ELISA, are required to investigate large numbers of monozygotic/dizygotic twins. Although the lectin-antibody ELISA that we recently developed (8) may be a suitable tool for large-scale analysis of IgG oligosaccharides, it is difficult to evaluate the normal levels of $\operatorname{IgG}$ agalactosylation using this method.

To summarize, although the ABO blood type is completely regulated by genetic factors, our results indicated that $\operatorname{IgG}$ oligosaccharides are more closely associated with environmental factors and genetic factors do not play a significant role. There are several reports available on the epigenetic regulation of glycosyltransferase genes $(8,11)$ and further studies are required to investigate the epigenetic and environmental factors affecting the agalactosylation of IgG.

\section{References}

1. Sox HC Jr and Hood L: Attachment of carbohydrate to the variable region of myeloma immunoglobulin light chains. Proc Natl Acad Sci USA 66: 975-982, 1970.

2. Takahashi N, Ishii I, Ishihara $\mathrm{H}$, et al: Comparative structural study of the N-linked oligosaccharides of human normal and pathological immunoglobulin G. Biochemistry 26: 1137-1144, 1987.

3. Mizuochi T, Taniguchi T, Shimizu A, et al: Structural and numerical variations of the carbohydrate moiety of immunoglobulin G. J Immunol 129: 2016-2020, 1982.

4. Parekh RB, Dwek RA, Sutton BJ, et al: Association of rheumatoid arthritis and primary osteoarthritis with changes in the glycosylation pattern of total serum IgG. Nature 316: 452-457, 1985.

5. Tomana M, Schrohenloher RE, Koopman WJ, et al: Abnormal glycosylation of serum IgG from patients with chronic inflammatory diseases. Arthritis Rheum 31: 333-338, 1988.

6. Bond A, Alavi A, Axford JS, et al: The relationship between exposed galactose and $\mathrm{N}$-acetylglucosamine residues on IgG in rheumatoid arthritis (RA), juvenile chronic arthritis (JCA) and Sjogren's syndrome (SS). Clin Exp Immunol 105: 99-103, 1996.

7. Shinzaki S, Iijima H, Nakagawa T, et al: IgG oligosaccharide alterations are a novel diagnostic marker for disease activity and the clinical course of inflammatory bowel disease. Am J Gastroenterol 103: 1173-1181, 2008.

8. Shinzaki S, Kuroki E, Iijima H, et al: Lectin-based immunoassay for aberrant IgG glycosylation as the biomarker for Crohn's disease. Inflamm Bowel Dis 19: 321-331, 2013.

9. Shinzaki S, Iijima H, Fujii H, et al: Altered oligosaccharide structures reduce colitis induction in mice defective in $\beta$-1,4-galactosyltransferase. Gastroenterology 142: 1172-1182, 2012 .

10. Parekh R, Isenberg D, Rook G, et al: A comparative analysis of disease-associated changes in the galactosylation of serum IgG. J Autoimmun 2: 101-114, 1989.

11. Kawamura YI, Toyota M, Kawashima R, et al: DNA hypermethylation contributes to incomplete synthesis of carbohydrate determinants in gastrointestinal cancer. Gastroenterology 135: 142-151, 2008. 